\title{
Engineering a Streptomyces coelicolor biosynthesis pathway into Escherichia coli for high yield triglyceride production
}

\author{
Santiago Comba, Martín Sabatini, Simón Menendez-Bravo, Ana Arabolaza* and Hugo Gramajo*
}

\begin{abstract}
Background: Microbial lipid production represents a potential alternative feedstock for the biofuel and oleochemical industries. Since Escherichia coli exhibits many genetic, technical, and biotechnological advantages over native oleaginous bacteria, we aimed to construct a metabolically engineered E. coli strain capable of accumulating high levels of triacylglycerol (TAG) and evaluate its neutral lipid productivity during high cell density fed-batch fermentations.
\end{abstract}

Results: The Streptomyces coelicolor TAG biosynthesis pathway, defined by the acyl-CoA:diacylglycerol acyltransferase (DGAT) Sco0958 and the phosphatidic acid phosphatase (PAP) Lpp $\beta$, was successfully reconstructed in an E. coli diacylglycerol kinase ( $d g k A)$ mutant strain. TAG production in this genetic background was optimized by increasing the levels of the TAG precursors, diacylglycerol and long-chain acyl-CoAs. For this we carried out a series of stepwise optimizations of the chassis by 1) fine-tuning the expression of the heterologous SCO0958 and Ipp $\beta$ genes, 2) overexpression of the S. coelicolor acetyl-CoA carboxylase complex, and 3) mutation of fadE, the gene encoding for the acyl-CoA dehydrogenase that catalyzes the first step of the $\beta$-oxidation cycle in $E$. coli. The best producing strain, MPS13/pET28-0958-ACC/PBAD-LPP $\beta$ rendered a cellular content of 4.85\% cell dry weight (CDW) TAG in batch cultivation. Process optimization of fed-batch fermentation in a 1-L stirred-tank bioreactor resulted in cultures with an $\mathrm{OD}_{600 \mathrm{~nm}}$ of 80 and a product titer of $722.1 \mathrm{mg} \mathrm{TAG} \mathrm{L}^{-1}$ at the end of the process.

Conclusions: This study represents the highest reported fed-batch productivity of TAG reached by a model non-oleaginous bacterium. The organism used as a platform was an E. coli BL21 derivative strain containing a deletion in the dgkA gene and containing the TAG biosynthesis genes from S. coelicolor. The genetic studies carried out with this strain indicate that diacylglycerol (DAG) availability appears to be one of the main limiting factors to achieve higher yields of the storage compound. Therefore, in order to develop a competitive process for neutral lipid production in E. coli, it is still necessary to better understand the native regulation of the carbon flow metabolism of this organism, and in particular, to improve the levels of DAG biosynthesis.

Keywords: TAG biosynthesis, Phosphatidate phosphatase, Escherichia coli, Oil production, Triacylglycerol

\section{Background}

Triacylglycerols (TAGs) are the most common lipid-based energy reserve in animals, plants, and eukaryotic microorganisms [1]. These neutral lipids, traditionally sourced from plant oils, have widespread applications in industry and healthcare, in addition to their main use in food and

\footnotetext{
* Correspondence: arabolaza@ibr-conicet.gov.ar; gramajo@ibr-conicet.gov.ar Microbiology Division, IBR (Instituto de Biología Molecular y Celular de Rosario), Consejo Nacional de Investigaciones Científicas y Técnicas, Facultad de Ciencias Bioquímicas y Farmacéuticas, Universidad Nacional de Rosario, Ocampo y Esmeralda (2000), Rosario, Argentina
}

feed purposes. In particular, about $20 \%$ of the plant oils produced today is utilized in certain applications of the oleochemical industry due to the lower downstream processing costs [2]. Furthermore, since the demand for oleochemicals is expected to grow, microbial production of free fatty acids (FAs) and FA-derived compounds such as TAGs, waxes, alkanes, or long-chain alcohols offers a great potential as an alternative to plant-derived products [1,3-5]. Compared to plant oils, microbial oils have many advantages; for example, they have a short life cycle, are less labor intensive, are not as affected by venue, season, 
and climate, and are easier to scale up [6]. The microbial production of oils also offers the possibility to reduce the competition with food feedstocks and the use of, for example, cellulose, lignin, hemicellulose, $\mathrm{CO}_{2}$, or other non-food carbon sources as raw material. Microorganisms that naturally synthesize TAG (like species of Rhodococcus, Mycobacterium, and Streptomyces genera) can reach high levels of this neutral lipid under specific growth conditions, as is the case of $R$. opaccus grown on gluconate medium which is capable of accumulating TAG accounting for up to $76 \%$ of the cell dry weight (CDW) [1,7]. Unfortunately, these microorganisms generally exhibit a rather slow growth rate and may require substantial and challenging genetic modifications for higher TAG productivity and/or for substrate utilization [8]. In this context, TAG production in Escherichia coli may become a valid alternative [9]. To date, a few recent studies have been published describing engineered pathways for this neutral lipid biosynthesis in E. coli $[8,10,11]$. These heterologous routes are based on the production and accumulation of the precursors, diacylglycerol (DAG) and fatty acyl-CoAs, which are esterified to synthesize TAG by the activity of a diacylglycerol acyltransferase (DGAT) (Figure 1). To raise the intracellular DAG concentration in E. coli, two alternatives have been described: 1 ) the overexpression of the native phosphatidylglycerol phosphate phosphatase PgpB [8], or the heterologous expression of the phosphatidate phosphatase Lpp $\beta$ from Streptomyces coelicolor [12]; and 2 ) the generation of a knockout mutant in the $E$. coli diacylglycerol kinase gene, $d k g A$. Deletion of $d g k A$ impairs the recycling of the DAG generated during the synthesis of membrane-derived oligosaccharides, thus leading to the accumulation of the DAG moiety $[13,14]$.

So far, TAG production in E. coli could be considered a feasible development if the engineering process could lead to a microorganism that accumulates TAG efficiently, while maintaining its fast growth properties [9]. Thus, the aim of this work was to construct a recombinant $E$. coli strain that could synthesize and store high levels of this neutral lipid. To do this we selected the two dedicated TAG biosynthesis enzymes from S. coelicolor, the phosphatidic acid phospatase Lpp $\beta$ and the acyl-CoA:diacylglycerol acyltransferase Sco0958, and followed a modular engineering strategy to optimize their expression levels in the heterologous host. Further genetic modifications introduced in the TAG producer strain allowed us to obtain an E. coli capable of accumulating the highest levels of TAG reported for fed-batch fermentations.

\section{Results and discussion}

Impact of the S. coelicolor PAP activity in TAG

biosynthesis in E. coli

In previous work Comba et al. demonstrated that the heterologous co-expression of the S. coelicolor DGAT

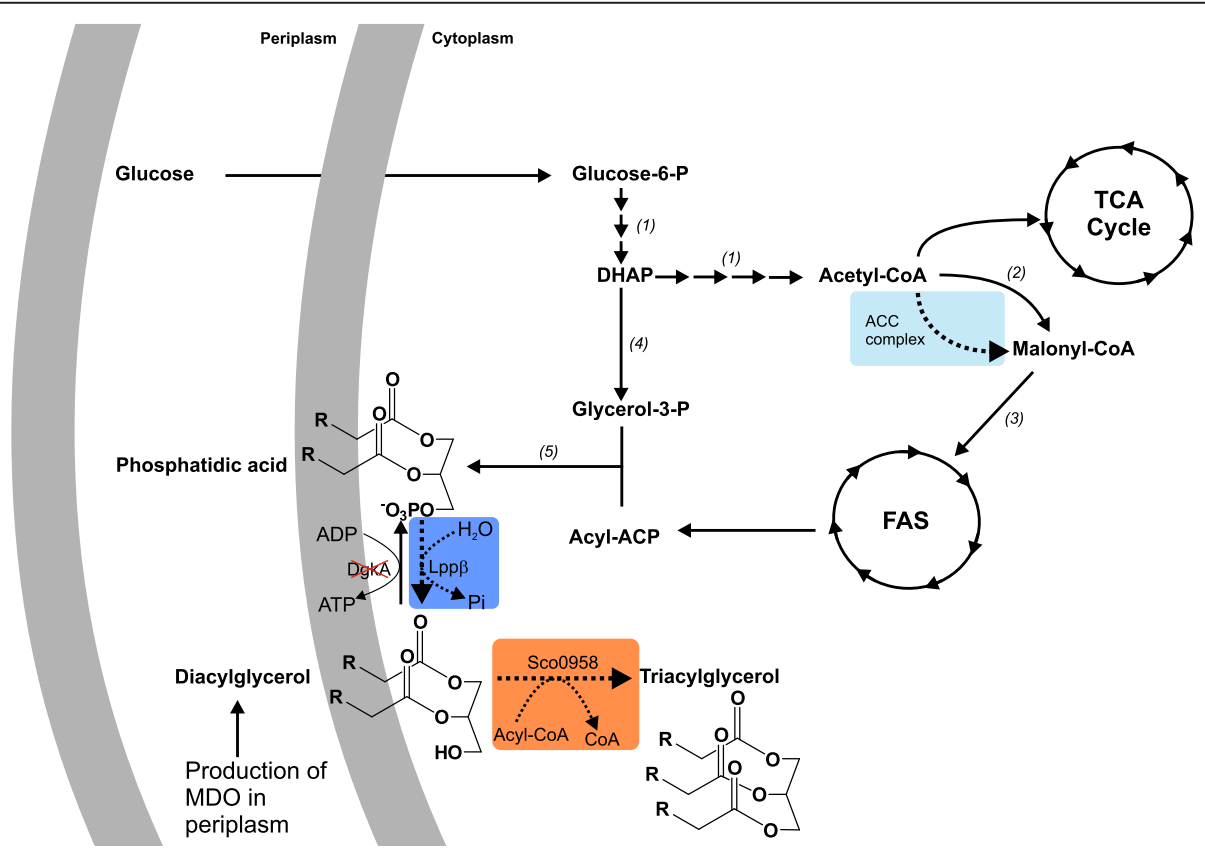

Figure 1 Engineering E. coli for TAG biosynthesis. Heterologous proteins expressed in E. coli are highlighted in color, and the knockout of the E. coli DgkA is labeled with a red cross. Sco0958, Lpp $\beta$, and ACC correspond to diacylglycerol acyltransferase, phosphatidic acid phosphatase, and acetyl-CoA carboxylase enzymes from S. coelicolor, respectively. (1) Glycolytic pathway; (2) acetyl-CoA carboxylase; (3) malonyl-CoA:ACP transacylase; (4) glycerol-3-P dehydrogenase; (5) glycerol-3-P and lysophosphatidic acid acyltransferases; DHAP, dihydroxyacetone phosphate; FAS, fatty acid synthase; TCA, tricarboxylic acid; MDO, membrane-derived oligosaccharide. 
(acyl-CoA:diacylglycerol acyltransferase) Sco0958 and the PAP (phosphatidic acid phosphatase) Lpp $\beta$, in an $E$. coli BL21 (DE3) strain leads to the production of TAG, while the sole expression of Sco0958 is unable to induce accumulation of this neutral lipid in the same genetic background [12]. On the other hand, Arabolaza et al. showed that the expression of the DGAT Sco0958 in an $E$. coli strain containing a deletion in the $d g k A$ gene is sufficient to induce TAG accumulation [15], suggesting that DAG is the limiting metabolite for TAG biosynthesis in E. coli. To test if DAG was still a limiting factor in a $d g k A$ background, we evaluated the impact of $\operatorname{Lpp} \beta$ in TAG biosynthesis in a BL21 $\Delta d g k A$ strain - named strain MPS11 - in the presence of Sco0958. To do this we transformed the MPS11 strain with plasmids PBAD0958, containing the $S C O 0958$ gene under the $\mathrm{P}_{\mathrm{BAD}}$ promoter, and pET28-LPP $\beta$, which contains the $\operatorname{lpp} \beta$ gene under the T7 promoter. As shown in Figure 2, the sole expression of SCO0958 in the MPS11 strain was sufficient to promote accumulation of significant levels of TAG (Figure 2, lane 2). However, co-expression of both the Sco0958 and Lpp $\beta$ enzymes increases TAG production by 1.6-fold (Figure 2, lane 4 versus lane 2). These results indicate that DAG availability is crucial for obtaining higher neutral lipid yields and that the PAP activity of Lpp $\beta$ does help to circumvent this bottleneck in the TAG biosynthesis pathway reconstructed in E. coli BL21.

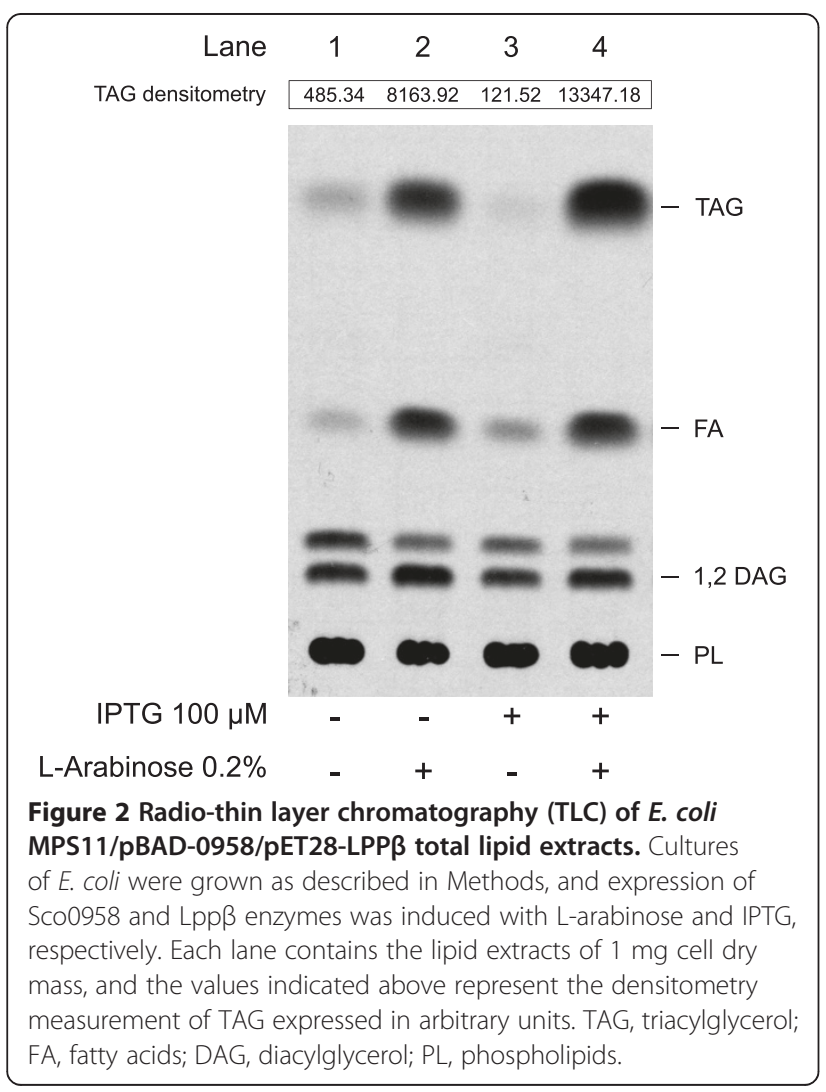

Interestingly, low levels of TAG biosynthesis were also detected under conditions of no induction of either $S C O 0958$ or $\operatorname{lpp} \beta$, or when only $\operatorname{lpp} \beta$ expression was induced (Figure 2, lanes 1 and 3, respectively); this low level of TAG production may occur by the leaky expression of SCO0958 from $\mathrm{P}_{\mathrm{BAD}}$ or, as suggested by Rotering and Raetz, by the existence of a hitherto unrecognized metabolism of minor neutral lipids biosynthesis in E. coli [16].

In this regard, the native $E$. coli phosphatidylglycerol phosphate phosphatase, PgpB, has been used as a surrogate PAP for TAG production [8]. Specifically, in a recent work Janssen and Steinbüchel described an E. coli K12 strain capable of producing $0.3 \%$ of the cellular dry mass of TAG by overexpressing PgpB and the fatty acylCoA synthase FadD from E. coli plus the DGAT AtfA from Acinetobacter baylyi [11]. Although this pathway was not reconstituted in a $d g k A$ background, making a direct comparison with our approach somehow difficult, it is appropriate to highlight that $\mathrm{PgpB}$ is a broad substrate spectrum enzyme, as demonstrated by its in vitro phosphatase activities towards phosphatidylglycerol, phosphatidic acid, lysophosphatidic acid, diacylglycerol pyrophosphate, and undecaprenyl pyrophosphate [17-19], while Lpp $\beta$ is a dedicated PAP involved in generating DAG for TAG biosynthesis in S. coelicolor [12].

\section{Fine-tuning TAG biosynthesis genes expression}

In order to optimize TAG production in our E. coli platform, we examined the use of alternative promoters, genes organization, and plasmid copy numbers to modulate the expression levels of SCO0958 and $\operatorname{lpp} \beta$ and to evaluate their impact in TAG biosynthesis. To do this, we constructed several expression vectors derived from plasmids pET28a or pBAD33 harboring either SCO0958 or $\operatorname{lpp} \beta$ as independent transcription units or as part of a bicistronic operon (Figure 3A). To analyze the levels of TAG produced by the individual expression systems, the corresponding plasmids were transformed into $E$. coli MPS11 and isolated clones of each of the four recombinant strains used for production studies in shake flasks. Figure 3B shows that the sole expression of SCO0958 from the strong $\mathrm{T} 7$ promoter notably increases TAG content in the cell respective to its expression from the $\mathrm{P}_{\mathrm{BAD}}$ promoter, demonstrating that under these conditions higher levels of the DGAT Sco0958 result in higher levels of TAG biosynthesis (Figure 3B, compare columns 1b and $2 \mathrm{~b})$. As expected, in all cases where the two genes of the system were induced, TAG accumulation was further improved, suggesting that the PAP Lpp $\beta$ increases the levels of DAG, which is otherwise a limiting metabolite of the system. Maximal production of TAG was reached by co-expressing SCO0958 from the strong T7 promoter and $\operatorname{lpp} \beta$ from $\mathrm{P}_{\mathrm{BAD}}$ (Figure 3B, column 2c). A western blot analysis of Sco0958 and Lpp $\beta$ expression for 
A

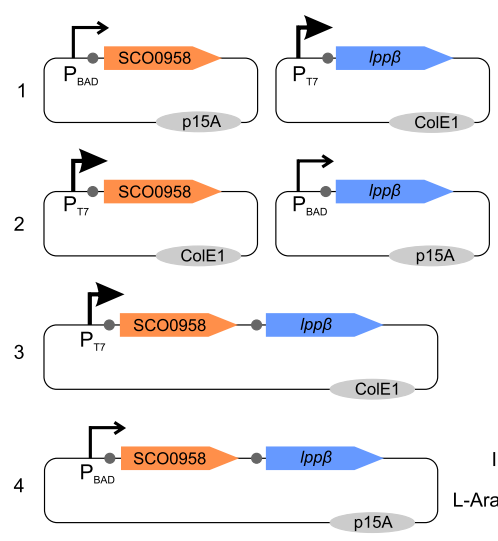

B

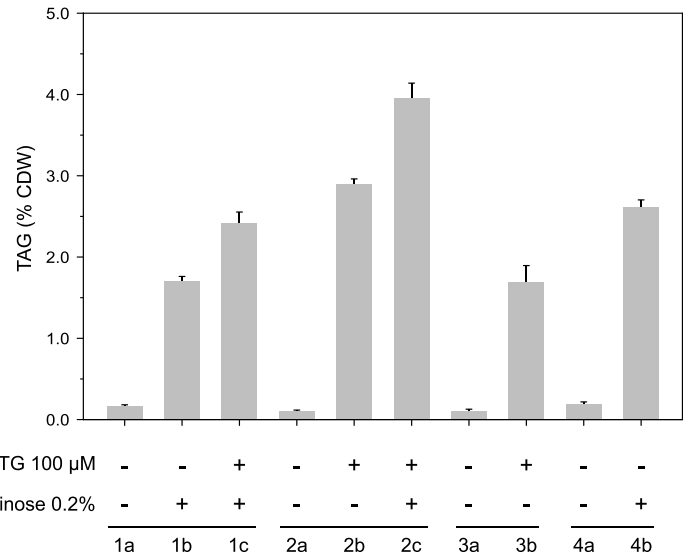

Figure 3 Optimization of TAG biosynthesis in the MPS11 strain. A) Schematic representation of the plasmid systems used for modulating SCO0958 and Ipp $\beta$ genes expression. The gray dot upstream of the target genes represents Shine-Dalgarno sequences and the size of the bended arrows, the relative strength of the inducible $\mathrm{P}_{\mathrm{T} 7}$ or $\mathrm{P}_{\mathrm{BAD}}$ promoters. B) Cultures of $E$. coli MPS11 containing the different expression systems (1 to 4) were grown as described in Methods section, and expression of the respective genes was induced with L-arabinose and/or isopropyl$\beta$-D-thiogalactopyranoside (IPTG). TAG content recovered from each of the cultures is expressed as percentage of cell dry weight mass (\% CDW).

each recombinant strain constructed indicates that construction 2, condition c of Figure 3 represents the optimum protein levels to reach maximal TAG accumulation (Additional file 1: Figure S1). Unexpectedly, the coupled expression of the SCO0958-lpp $\beta$ operon, either from $\mathrm{P}_{\mathrm{T} 7}$ or $\mathrm{P}_{\mathrm{BAD}}$ promoters, was accompanied by a medium to low accumulation of TAG, suggesting that in either case the relationship between Sco0958 and Lpp $\beta$ was not optimal (Figure 3B, columns $3 \mathrm{~b}$ and $4 \mathrm{~b}$ ). In these single-plasmid systems, the levels of expression of both enzymes are lower compared to the situation where the SCO0958 and $\operatorname{lpp} \beta$ genes are located in two different plasmids (Additional file 1: Figure S1), leading to a suboptimal activity of the pathway. Overall, these results support the notion that expression of SCO0958 and $\operatorname{lpp} \beta$ has to be finely modulated in order to achieve optimal levels of TAG biosynthesis. Further studies will be necessary to better understand the basis of the transcriptiontranslation dynamics of these genes in both expression systems.

Curiously, although the $\mathrm{P}_{\mathrm{BAD}}$ promoter is weaker than $\mathrm{P}_{\mathrm{T} 7}$ and the p15A origin of replication has a lower copy number than ColE1, the Lpp $\beta$ protein levels in condition $2 \mathrm{c}$ are slightly higher than in condition 1c (Additional file 1: Figure S1). Moreover, when both proteins are expressed, the Sco0958 protein titer decreases compared to the situation where it is induced alone. This effect could be due to the high metabolic burden imposed on the cell when two, instead of one, proteins are expressed from strong promoters. In this sense, to further optimize the TAG-producing strain MPS11/pET28-0958/pBAD$\mathrm{LPP} \beta$, we evaluated the inducer dose-response to find the best L-arabinose concentration to reach maximal
TAG content. Cultures of the producer strain were grown in Luria-Bertani (LB) medium and induced with different concentrations of L-arabinose (Figure 4, lanes 5 to 8). TAG quantification carried out by LC-MS revealed that $0.1 \%$ of $\mathrm{L}$-arabinose was the inducer concentration that leads to the highest TAG content. Furthermore, to evaluate a possible contribution from the catabolism of L-arabinose in TAG production, we included a control with an empty pBAD33 plasmid instead of the pBADLPP $\beta$ vector. In these cultures, increasing concentrations of L-arabinose had no effect on the final TAG content (Figure 4, lanes 1 to 4 ).

In summary, the systematic studies carried out towards optimizing the expression levels of the specific TAG biosynthesis genes led us to identify the most suitable strain, MPS11/pET28-0958/pBAD-LPP $\beta$, and gene induction conditions, $100 \mu \mathrm{M}$ IPTG and $0.1 \% \mathrm{~L}$-arabinose, to reach a maximal TAG production of $4.15 \%$ CDW.

\section{Neutral lipid profile of engineered $E$. coli expressing Sco0958 or AtfA}

The AtfA enzyme from $A$. baylyi was the first member of the thereafter designated WS/DGAT prokaryotic family of proteins [20]. Heterologous expression of this enzyme has been used for the production of fatty acid methyl esters, waxes, and TAG in E. coli $[3,8,21]$. In order to specifically evaluate the TAG biosynthesis capabilities of AtfA and compare it with our producer strain containing Sco0958 as a DGAT, we cloned the atfA gene in the pET28 expression vector, generating plasmid pET28-AtfA, and transformed it into an MPS11 derivative strain harboring the pBAD-LPP $\beta$ plasmid. As shown in Figure 5, AtfA expression led to a significant production of TAG, 


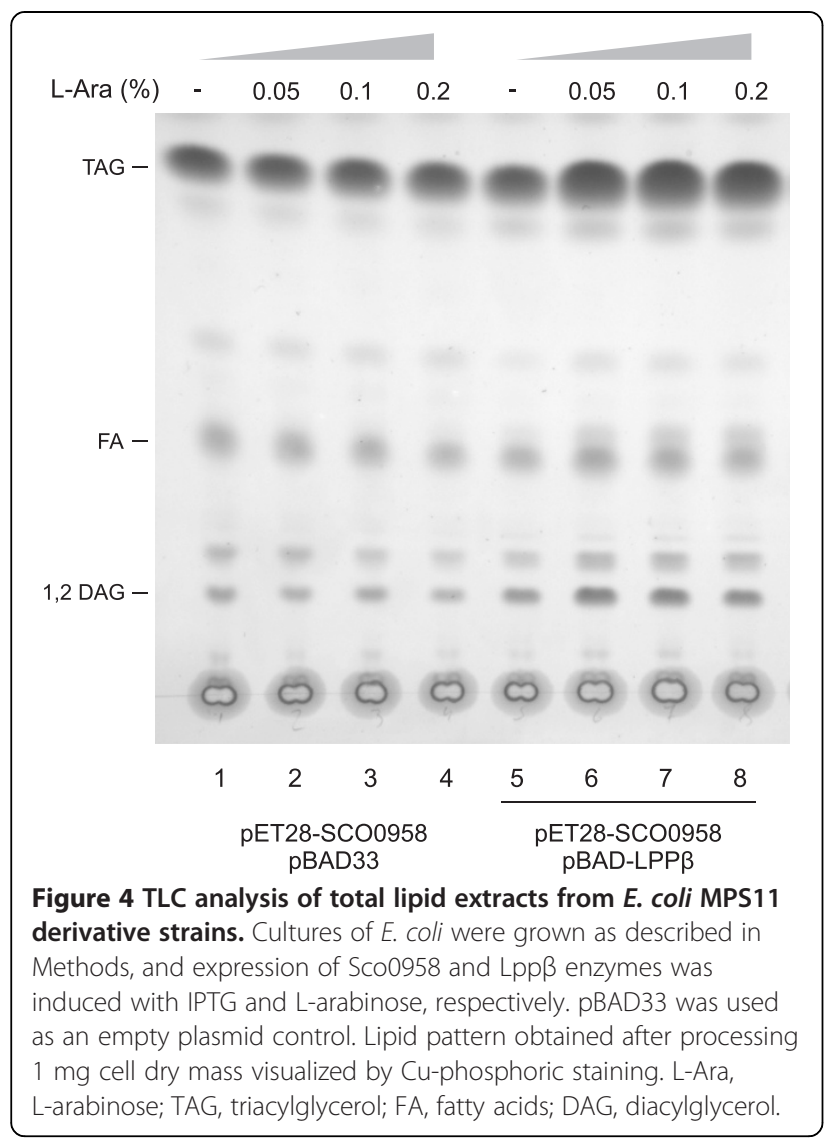

but also of an additional compound identified as wax esters by their retention factor on the TLC plates. This result agrees with previous reports where AtfA is confirmed to be capable of synthesizing butyl esters in E. coli, even in the absence of a supplemented alcohol [3]. Moreover, the levels of TAG achieved with AtfA were 10.6\% lower than the ones obtained with Sco0958. Although expression of AtfA could be optimized to increase TAG titers, these results suggest that Sco0958 has a higher specificity than AtfA for TAG synthesis and, therefore, Sco0958 appears to be a better candidate for TAG production in E. coli.

\section{Metabolic engineering further improves TAG accumulation}

To further improve TAG titers in our recombinant strain, we followed a classic metabolic engineering approach to enhance the pools of substrates of the TAG biosynthesis enzymes. To increase fatty acyl-CoA availability, we proceeded to engineer the fatty acid oxidation pathway of $E$. coli through the overexpression of $f a d D$ and the disruption of fadE. FadD activates free fatty acids into the metabolically active acyl-CoA thioesters [22], and FadE is an acyl-CoA dehydrogenase that catalyzes the first step of the $\beta$-oxidation cycle, the oxidation of an acyl-CoA to a 2-enoyl-CoA [23]. The co-expression of Sco0958 and

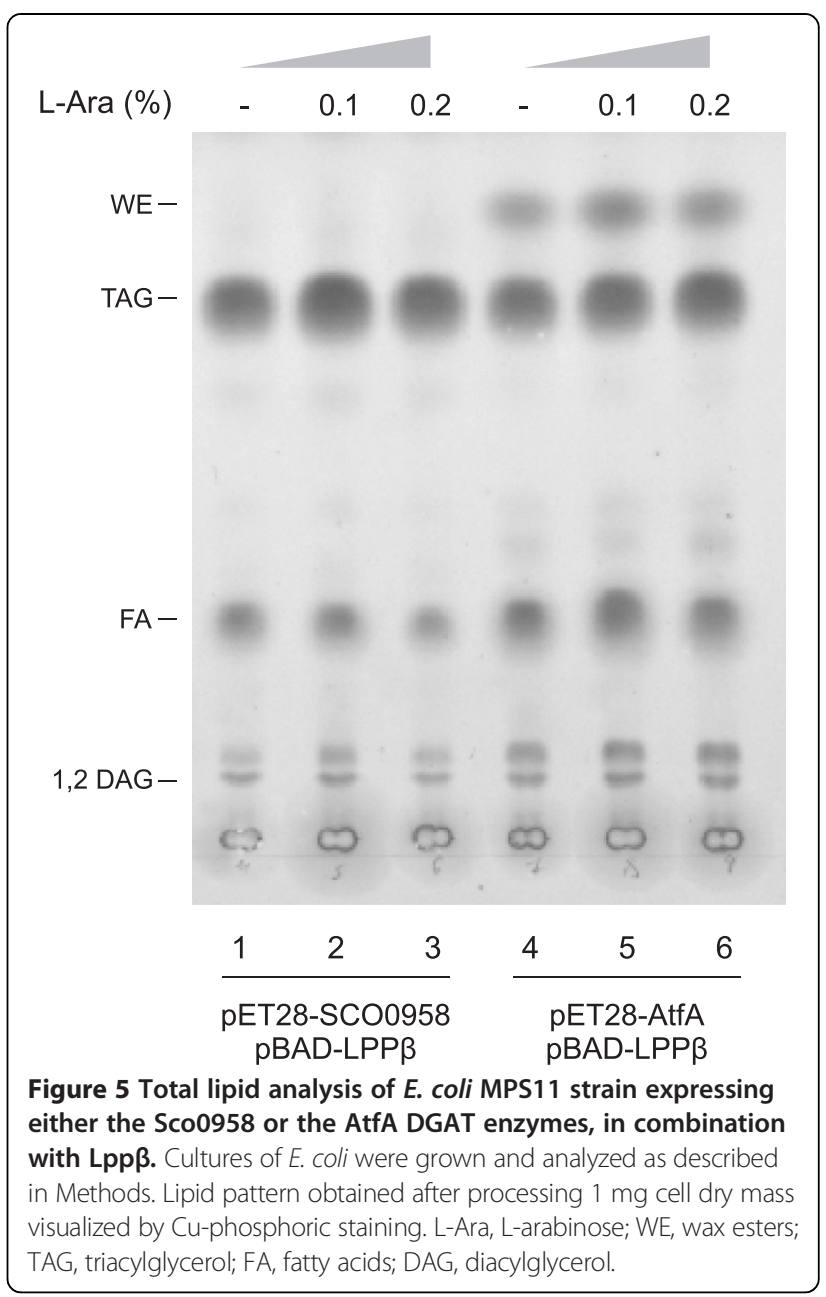

Lpp $\beta$ enzymes in a BL21 $\Delta d g k A \Delta f a d E$ strain - named the MPS13 strain - led to a 9\% higher accumulation of TAG compared with its parental strain MPS11. Unexpectedly, overexpression of FadD in the $\triangle d g k A \Delta f a d E$ genetic background did not further increase the levels of TAG (Additional file 1: Table S1). This result suggests that under the conditions tested the native FadD activity is sufficient to activate the available FAs to their respective acyl-CoAs.

In order to further optimize TAG production in E. coli, we decided to overexpress proteins involved in key steps of the central carbon metabolism that could be working as bottlenecks in the carbon flow towards TAG biosynthesis. For this purpose we selected the MPS13/pBAD-LPP $\beta$ strain and transformed it with several pET28-based vectors for the co-expression of Sco0958 with the following selected targets: 1) GpsA, a glycerol 3-phosphate (G3P) dehydrogenase from $S$. coelicolor, which catalyzes the generation of G3P from the glycolysis intermediate dihydroxyacetone phosphate. This enzyme was selected based on previous results that showed that expression of a Saccharomyces cerevisiae G3P dehydrogenase in Brassica 
napus raised their seed oil content by $40 \%$ [24]; 2) a second copy of SCO0958; 3) FadR, the dual transcriptional regulator of fatty acid metabolism in E. coli $[25,26]$. FadR is a repressor of the $\beta$-oxidation genes and an activator of the fatty acid biosynthesis genes $f a b A$ and $f a b B$. The selection of FadR was based on previous results where it was shown that its overexpression in E. coli led to a significant increase in fatty acid content [27]; 4) The acetyl-CoA carboxylase complex (ACC) from S. coelicolor, constituted by three subunits AccA2, AccB, and AccE, which generates the malonyl-CoA utilized for fatty acid chain elongation $[28,29]$. The analysis of TAG accumulation in each of the derivatives strains containing the combination of genes described above indicated that neither gpsA, nor the extra copies of SCO0958 or fadR improved the levels of TAG obtained with the original system (4.52\% CDW in MPS13/pET28-0958/pBAD-LPP $\beta$, Additional file 1: Table S1). However, expression of the $S$. coelicolor ACC complex led to a $7.3 \%$ increase in TAG production, reaching a TAG content of 4.85\% CDW (Additional file 1: Table S1). ACC complex overexpression is expected to increase FA synthesis, thus raising the levels of FAderivative lipids. In this context, ACC enzymes could have a double role in TAG synthesis precursor supply, since it would increase the availability of DAG through the increase of PA - and/or of the fatty acylCoA precursors.

Several metabolic engineering efforts have been carried out in E. coli in order to improve FA production, as they are potential precursors for FA-derived hydrocarbons $[4,5,9,10,27,30-37]$. However, little has been done in this host to channel these FAs towards TAG biosynthesis. In this sense, Lin et al. constructed an E. coli BL21 derivative strain carrying multiple combinations of deletions in genes involved in central carbon flux [10]. Although they successfully increased FA availability in some of their mutant strains, they did not obtain a concomitant increase in
TAG content when the TAG biosynthesis enzymes were overexpressed [10]. These results confirm that additional bottlenecks must be overcome to achieve higher TAG titers. Interestingly, Lin et al. did not use a PAP enzyme to improve DAG availability in their hosts, which according to our results is the main limiting metabolite for TAG biosynthesis.

\section{Fed-batch cultivation for high yield TAG production}

Based on the results obtained in our batch culture studies, we selected the MPS13/pET28-0958-ACC/pBADLPP $\beta$ strain for the optimization of TAG production in high cell density fermentations. Before initiating these studies, we introduced one additional mutation in the producing strain in order to prevent L-arabinose metabolization and thus avoid permanent feeding of the inducer; to do this we knocked out the complete $\operatorname{araBAD}$ operon in MPS13, giving rise to the BL21 $\triangle d g k A \triangle f a d E$ $\triangle a r a B A D$, named the MPS15 strain. Fed-batch cultures were performed in minimal medium, using glucose as the carbon source, at $37^{\circ} \mathrm{C}$. Heterologous protein production was induced by addition of $1 \mathrm{mM}$ IPTG and L-arabinose $0.1 \%$ at $\mathrm{OD}_{600 \mathrm{~nm}}$ about 60 after shifting the temperature of the process to $23^{\circ} \mathrm{C}$ to improve protein solubility. Glucose was kept limiting throughout the process in order to avoid overflow of the tricarboxylic acid (TCA) cycle and the concomitant production of acetate. Dissolved oxygen was maintained above $40 \%$ to prevent anaerobic fermentation of the carbon source. Samples were taken periodically for neutral lipids analysis.

Figure 6 shows the progression of the fed-batch cultures carried out in this study. The complete process took $68 \mathrm{~h}$, and at the end of the fermentation, the MPS15/pET28-0958-ACC/pBAD-LPP $\beta$ strain was able to synthesize $722.1 \mathrm{mg}$ TAG $\mathrm{L}^{-1}$, reaching a cell TAG content of $2.83 \% \mathrm{CDW}$. Under these conditions, the volumetric productivity was $10.54 \mathrm{mg}$ TAG $\mathrm{L}^{-1} \mathrm{~h}^{-1}$ at the

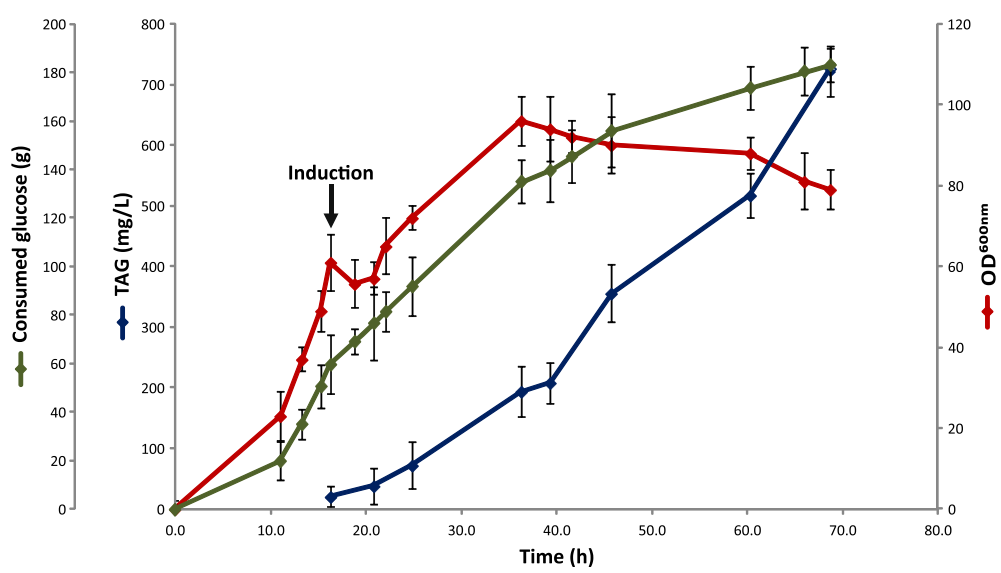

Figure 6 Fed-batch cultivation of MPS15/pET28-0958-ACC/pBAD-LPP $\beta$ strain. E. coli strain was cultured as described in Methods, and samples were taken periodically for OD, glucose, and neutral lipids analysis. 
end of the process. Furthermore, the FA composition of the resulting E. coli single cell oil was also analyzed and is described in Additional file 1: Table S2. The most abundant triglyceride species reflect the composition of total E. coli $\mathrm{FA}$, as they are composed mainly of saturated and monounsaturated C16 and C18 FAs. Similar results were reported by Rucker et al. by analyzing the FA composition of E. coli TAG grown in batch cultures [8].

To our knowledge, the only other study where TAG production in E. coli was evaluated in fed-batch fermentors is that from Janssen and Steinbüchel [11]. In their work the authors used an E. coli $\mathrm{K} 12$ derivative strain that reached a final OD $_{600 \mathrm{~nm}}$ of 13.2 after $68 \mathrm{~h}$ and produced $532 \mathrm{mg} \mathrm{L}^{-1}$ TAG, with a maximal TAG content of 8.5\% CDW under limited nitrogen conditions [11].

Although the maximal TAG content of strain MPS15/ pET28-0958-ACC/pBAD-LPP $\beta$ obtained during the fermentation process was significantly lower than the ones obtained in batch assays, the cultures grown in the reactors were still able to produce the highest TAG titers reported for E. coli until now. It is difficult to predict the cause(s) of this striking difference in TAG accumulation between the two different growth systems, although we could speculate that the use of minimal medium in the reactors, instead of LB, could account for such a difference. However, we also observed that batch cultures of the selected strain carried out in minimal medium supplemented with $5 \mathrm{~g} \mathrm{~L}^{-1}$ glucose - which is below the catabolite repression threshold - have the same production levels as the batch cultures grown in LB medium (data not shown). Therefore, other bottlenecks or limitations appear to exist during fermentation that do not allow higher accumulation of the neutral lipid. We speculate that further improvements or optimization of the fermentation process should let us reach the production levels that were achieved in batch cultures.

\section{Conclusions}

In this work we described the successful reconstruction of the S. coelicolor TAG biosynthesis pathway in the model heterologous host $E$. coli. By genetically engineering the $E$. coli producer strain, we improved the final TAG content up to $4.85 \% \mathrm{CDW}$ in batch cultures. Additionally, the fed-batch microbial fermentation was also optimized, achieving a maximum yield of $722.1 \mathrm{mg} \mathrm{TAG}^{-1}$ and a volumetric productivity of $10.54 \mathrm{mg}$ TAG $\mathrm{L}^{-1} \mathrm{~h}^{-1}$. These results represent the highest reported fed-batch productivity of TAG reached by a non-oleaginous heterologous bacterium. However, the titer of TAG produced by this recombinant $E$. coli is still low compared with those of natural oleaginous producers such as Rhodococcus sp. Considering the productivities of TAG reported for fed-batch fermentations of Rhodococcus opaccus, much basic research is needed in the E. coli system in order to efficiently convert the high levels of FA that this organism can produce into higher concentrations of TAG or of other high-value hydrocarbons of industrial interest.

\section{Methods}

\section{Media and growth conditions}

E. coli strains were routinely grown either on solid or in liquid $\mathrm{LB}$ medium at $37^{\circ} \mathrm{C}$ and supplemented when needed with the following antibiotics: $100 \mu \mathrm{g} \mathrm{mL}^{-1}$ ampicillin (Ap), $50 \mu \mathrm{g} \mathrm{mL}^{-1}$ kanamycin $(\mathrm{Km})$, or $20 \mu \mathrm{g} \mathrm{mL}$ chloramphenicol $(\mathrm{Cm})$. For auxotrophy tests, M9 mineral media supplemented with either $0.2 \%$ glucose, $0.2 \%$ L-arabinose, or $0.1 \%$ oleate $/ 0.4 \%$ Brij 58 were used. For solid media, $1.5 \%$ agar was added before autoclaving.

The minimal medium for fed-batch fermentation consisted of $0.5 \mathrm{~g} \mathrm{~L}^{-1}$ yeast extract, $20.8 \mathrm{~g} \mathrm{~L}^{-1} \mathrm{KH}_{2} \mathrm{PO}_{4}, 3 \mathrm{~g} \mathrm{~L}^{-1}$ $\left(\mathrm{NH}_{4}\right)_{2} \mathrm{HPO}_{4}$, and $4 \mathrm{~g} \mathrm{~L}^{-1} \mathrm{NaH}_{2} \mathrm{PO}_{4}$. After autoclaving, it was supplemented with $50 \mu \mathrm{g} \mathrm{mL}^{-1} \mathrm{Km}, 20 \mu \mathrm{g} \mathrm{mL}{ }^{-1} \mathrm{Cm}$, $1.5 \mathrm{~mL}$ of trace element solution per liter, and the corresponding amount of carbon source solution. The trace element solution contained $50 \mathrm{mM} \mathrm{FeCl} 3,20 \mathrm{mM} \mathrm{CaCl}_{2}$, $10 \mathrm{mM} \mathrm{ZnSO}$, $10 \mathrm{mM} \mathrm{MnCl}$, $2 \mathrm{mM} \mathrm{CoCl} 2,2 \mathrm{mM}$ $\mathrm{CuCl}_{2}, 2 \mathrm{mM} \mathrm{NiCl}_{2}, 2 \mathrm{mM} \mathrm{H}_{3} \mathrm{BO}_{3}, 2 \mathrm{mM} \mathrm{Na}_{2} \mathrm{MoO}_{4}$, and $2 \mathrm{mM} \mathrm{Na} 2 \mathrm{SeO}_{3}$. The carbon source solution used contained $600 \mathrm{~g} \mathrm{~L}^{-1}$ glucose and $15 \mathrm{~g} \mathrm{~L}^{-1} \mathrm{MgSO}_{4} \cdot 7 \mathrm{H}_{2} \mathrm{O}$.

\section{Strain construction}

All the strains used in this study are listed in Table 1. The E. coli MPS11 strain was constructed by P1 transduction of the $d g k A:: K m$ marker from the JW4002 strain [38] to BL21 and subsequent FLP recombinase-mediated excision of the kanamycin cassette as described by Datsenko and Wanner [39]. The MPS13 strain was constructed by P1 transduction of the fadE::Km marker from the JW5020 strain [38] to MPS11 and subsequent FLP recombinase-mediated excision of the kanamycin cassette [39]. The MPS14 strain was constructed by the one-step inactivation of $E$. coli chromosomal genes described by Datsenko and Wanner [39]. For this, araBAD_F/araBAD_R oligonucleotides were used to amplify the $\mathrm{Km}$ cassette of the pKD13 plasmid. The resulting DNA fragment was used to transform MPS13/pKD46 electrocompetent cells to replace $\operatorname{araBAD}$ genes. The kanamycin resistance cassette of strain MPS14 was deleted by FLP recombinase-mediated excision, yielding the MPS15 strain [39]. Deletions of $d g k A$ and $f a d E$ genes were checked by PCR using dgkA_F/R and fadE_F/R primer pairs. Deletion of araBAD genes was checked by PCR using ara_check_F/ara_check_R and araA_wt_F/ ara_check_R primer pairs. Knockout of $f a d E$ and $a r a B A D$ genes was also confirmed by growing the strains in M9 plates with glucose, arabinose, or oleate as sole carbon sources. 
Table 1 Strains and plasmids

\begin{tabular}{|c|c|c|}
\hline Strain or plasmid & Description & Reference \\
\hline \multicolumn{3}{|l|}{ Strains } \\
\hline $\mathrm{DH} 5 \mathrm{a}$ & E. coli K12 F lacU169 (\$80lacZUM15) endA1 recA1 hsdR17 deoR supE44 thi-1 12 gyrA96 relA1 & [40] \\
\hline BL21 (DE3) & E. coli F ompT gal dcm lon hsdS $\left(r_{B}^{-} m_{B}^{-}\right) \lambda(D E 3)$ & Novagen \\
\hline MPS10 & BL21 (DE3) $\triangle d g k A:: K m ; \mathrm{Km}^{\mathrm{R}}$ & This study \\
\hline MPS11 & BL21 (DE3) $\triangle d g k A$ & This study \\
\hline MPS12 & BL21 (DE3) $\triangle d g k A \triangle f a d E:: K m ; \mathrm{Km}^{R}$ & This study \\
\hline MPS13 & BL21 (DE3) $\triangle d g k A \triangle f a d E$ & This study \\
\hline MPS14 & BL21 (DE3) $\triangle d g k A \triangle f a d E \triangle a r a B A D \because: K m ; \mathrm{Km}^{R}$ & This study \\
\hline MPS15 & $\mathrm{BL} 21$ (DE3) $\triangle d g k A \triangle \mathrm{fadE} \triangle \mathrm{araBAD}$ & This study \\
\hline \multicolumn{3}{|l|}{ Plasmids } \\
\hline pET28a & Vector for expression of $\mathrm{N}$-terminal His-tagged proteins under the strong $\mathrm{T} 7$ promoter; $\mathrm{Km}^{\mathrm{R}}$ & Novagen \\
\hline pBAD33 & Vector for recombinant protein expression under the control of the $P_{B A D}$ promoter; $\mathrm{Cm}^{\mathrm{R}}$ & [41] \\
\hline pKD13 & Template plasmid for amplification of the FRT-flanked kanamycin cassette; $A p^{R} \mathrm{Km}^{R}$ & [39] \\
\hline pKD46 & Temperature-sensitive replication plasmid for Red recombinase expression; $A p^{R}$ & [39] \\
\hline pCP20 & Temperature-sensitive replication plasmid for thermal induction of FLP synthesis; $\mathrm{Cm}^{\mathrm{R}} \mathrm{Ap}^{\mathrm{R}}$ & [42] \\
\hline pCR ${ }^{\circledast}$-Bluntll-TOPO & Vector used for cloning of blunt PCR products; $\mathrm{Km}^{\mathrm{R}}$ & Invitrogen \\
\hline pBAD0958 & pBAD33 carrying the $S C 00958^{\text {His }}$ gene under the control of $P_{B A D}$ promoter; $\mathrm{Cm}^{\mathrm{R}}$ & [15] \\
\hline pET28-LPPß & pET28 carrying the $\mathrm{SCO} 1753^{\mathrm{His}}$ gene under the control of $\mathrm{T} 7$ promoter; $\mathrm{Km}^{\mathrm{R}}$ & [12] \\
\hline PBAD-LPPB & pBAD33 carrying the $\mathrm{SCO} 1753^{\mathrm{His}}$ gene under the control of $P_{B A D}$ promoter; $\mathrm{Cm}^{\mathrm{R}}$ & [12] \\
\hline pTR257 & pET28 carrying the $\mathrm{SCO} 0958^{\mathrm{His}}$ gene under the control of T7 promoter; $\mathrm{Km}^{\mathrm{R}}$ & {$[15]$} \\
\hline pET28-0958- LPP $\beta$ & pET28 carrying the $\mathrm{SCO} 0958^{\mathrm{His}}-\mathrm{SCO} 1753^{\mathrm{His}}$ operon under the control of $\mathrm{T7}$ promoter; $\mathrm{Km}^{\mathrm{R}}$ & This study \\
\hline pBAD-0958- LPPß & pBAD33 carrying the $\mathrm{SCO} 0958^{\mathrm{His}}-\mathrm{SCO} 1753^{\mathrm{His}}$ operon under the control of $P_{B A D}$ promoter; $\mathrm{Cm}^{\mathrm{R}}$ & This study \\
\hline pET28-AtfA & pET28 carrying the atf $A^{H i s}$ gene under the control of $\mathrm{T} 7$ promoter; $\mathrm{Km}^{\mathrm{R}}$ & This study \\
\hline pET28-FadD & pET28 carrying the fadD ${ }^{\text {His }}$ gene under the control of T7 promoter; $\mathrm{Km}^{\mathrm{R}}$ & This study \\
\hline pET28-0958- FadD & pET28 carrying the $\mathrm{SCO} 0958^{\mathrm{His}}$-fadD $\mathrm{D}_{\mathrm{His}}$ operon under the control of $\mathrm{T} 7$ promoter; $\mathrm{Km}^{\mathrm{R}}$ & This study \\
\hline pCC01 & pET28 carrying the accA2 $2^{\text {His }}$ gene under the control of $\mathrm{T} 7$ promoter; $\mathrm{Km}^{\mathrm{R}}$ & [43] \\
\hline pET28-AccA2-AccE & pET28 carrying the $a c c A 2^{\mathrm{His}}$-accE $E^{\mathrm{His}}$ operon under the control of $\mathrm{T} 7$ promoter; $\mathrm{Km}^{\mathrm{R}}$ & This study \\
\hline pET28-AccA2-AccE-AccB & pET28 carrying the $a c c A 2^{\mathrm{His}}$-acc $E^{\mathrm{His}}$-accB $B^{\mathrm{His}}$ operon under the control of $\mathrm{T} 7$ promoter; $\mathrm{Km}^{\mathrm{R}}$ & This study \\
\hline pET28-0958-ACC & pET28 carrying the $\mathrm{SCO} 0958^{\mathrm{His}}$-accA2 ${ }^{\mathrm{His}}$-acc $\mathrm{H}^{\mathrm{His}}$-acc $\mathrm{B}^{\mathrm{His}}$ operon under the control of $\mathrm{T} 7$ promoter; $\mathrm{Km}^{\mathrm{R}}$ & This study \\
\hline pET28-GpsA & pET28 carrying the $g p s A^{\text {His }}$ gene under the control of T7 promoter; $\mathrm{Km}^{\mathrm{R}}$ & This study \\
\hline pET28-0958-GpsA & pET28 carrying the $S C 00958^{\mathrm{His}}$-gps $A^{\mathrm{His}}$ operon under the control of $\mathrm{T7}$ promoter; $\mathrm{Km}^{\mathrm{R}}$ & This study \\
\hline pET28-FadR & pET28 carrying the fadR $R^{H i s}$ gene under the control of $\mathrm{T} 7$ promoter; $\mathrm{Km}^{\mathrm{R}}$ & This study \\
\hline pET28-0958-FadR & pET28 carrying the $\mathrm{SCO} 0958^{\mathrm{His}}$-fadR $R_{\text {His }}$ operon under the control of T7 promoter; $\mathrm{Km}^{\mathrm{R}}$ & This study \\
\hline pET28-0958-0958 & pET28 carrying the $\mathrm{SCO} 0958^{\mathrm{His}}-\mathrm{SCO} 0958^{\mathrm{His}}$ operon under the control of T7 promoter; $\mathrm{Km}^{\mathrm{R}}$ & This study \\
\hline
\end{tabular}

\section{Plasmid construction}

All the plasmids used in this study are listed in Table 1. The oligonucleotides used for PCR are listed in Table 2. The pET28-0958-LPP $\beta$ vector was constructed by ligation of the XbaI/HindIII digestion fragment of pET28-LPP $\beta$ into SpeI/HindIII restriction sites of pTR257. The XbaI/ HindIII digestion fragment was then cloned in XbaI/ HindIII sites of pBAD33, yielding plasmid pBAD0958-LPP $\beta$.

The atfA gene [GenBank:2879218] was amplified by PCR from Acinetobacter sp. ADP1 genomic DNA using the
atfA_F/atfA_R primer pair. The fadD [GenBank:8182496] and $\mathrm{fadR}$ [GenBank:8181871] genes were amplified by PCR from $E$. coli BL21 genomic DNA using the fadD_F/fadD_R and fadR_F/fadR_R primer pairs, respectively. The gpsA [GenBank:1101000], accB [GenBank:1100975], and $a c c E$ [GenBank:1100976] genes were amplified by PCR from S. coelicolor genomic DNA using the gpsA_F/gpsA_R, accB_F/accB_R and accE_F/accE_R primer pairs, respectively. The resulting PCR products were cloned in the $\mathrm{pCR}^{\circ}$-BluntII-TOPO vector and checked by DNA 
Table 2 PCR oligonucleotides

\begin{tabular}{|c|c|c|}
\hline Name & Sequence $\left(5^{\prime}-3^{\prime}\right)$ & Reference \\
\hline dgkA_F & AGGATCTGCCGGAATAGACTTGCTT & This study \\
\hline dgkA_R & AGCGCCTTCAGATGTTCTTCAGCC & This study \\
\hline fadE_F & CAAAAGCGAGAAGTACGGGCAGGTG & This study \\
\hline fadE_R & GCTITCGATTGATGGTAAAACGGTG & This study \\
\hline araBAD_F & $\begin{array}{l}\text { CTCGATTITGGCAGTGATTCTGTGCGAGCTIT } \\
\text { GGCGGTGGCTGTCAAACATGAGAATTAA }\end{array}$ & This study \\
\hline $\operatorname{araBAD} \_\mathrm{R}$ & $\begin{array}{l}\text { CCGTAATATGCCTTCGCGCCATGCTTACGCA } \\
\text { GATAGTGTTGTGTAGGCTGGAGCTGCTTC }\end{array}$ & This study \\
\hline ara_check_F & TAAACGAGTATCCCGGCAGCAGGGG & This study \\
\hline ara_check_R & CGGGAATAAACGCCACGGACTCTTG & This study \\
\hline araA_wt_F & AAGTGTATTACGGGTTTCGTCGCTA & This study \\
\hline atfA_F & CATATGCGCCCATTACATCCGATTG & This study \\
\hline atfA_R & ACTAGTCCTITAGTTTTATCTGATA & This study \\
\hline fadD_F & GCTAGCAAGAAGGTTTGGCTTAACC & This study \\
\hline fadD_R & ACTAGTCTCAGGCTITATTGTCCAC & This study \\
\hline gpsA_F & CATATGAGCAAGCCGGTCAAGGCGG & This study \\
\hline gpsA_R & ACTAGTGCGGGTTGCGCGGGGGGTC & This study \\
\hline fadR_F & CATATGGTCATTAAGGCGCAAAGCC & This study \\
\hline fadR_R & ACTAGTATGGGAAATCTGTAAAAAC & This study \\
\hline accB_F & CATATGACCGTITGGATGAGGCGC & This study \\
\hline accB_R & ACTAGTTCACTGCGGCGGGTTGCCG & This study \\
\hline accE_F & CATATGTCCCCTGCCGACATCCGCG & This study \\
\hline accE_R & ACTAGTTCAGCGCCAGCTGTGCGGG & This stud) \\
\hline
\end{tabular}

Restriction sites are shown in italics. Homologous sequences to pKD13 plasmid are shown in bold.

sequencing (University of Maine DNA Sequencing Facility, Orono, ME, USA). The AtfA, FadR, GpsA, AccB, and AccE coding sequences were cloned in pET28a as NdeI/EcoRI digestion fragments, yielding plasmids pET28-AtfA, pET28-FadR, pET28-GpsA, pET28-AccB, and pET28-AccE, respectively. fadD was cloned as an NheI/EcoRI digestion fragment into pET28a, yielding plasmid pET28-FadD.

The pET28-AccA2-AccE plasmid was constructed by ligation of the $\mathrm{XbaI} / \mathrm{HindIII}$ digest of $\mathrm{pET} 28$-AccE into Spel/HindIII sites of pCC01. The XbaI/HindIII digestion fragment of pET28-AccB was then cloned into SpeI/ HindIII sites of pET28-AccA2-AccE, yielding plasmid pET28-AccA2-AccE-AccB. This plasmid carries the coding sequences for the complete acetyl-CoA carboxylase complex of S. coelicolor.

pET28a derivative vectors carrying the FadD, AccA2AccE-AccB, FadR, GpsA, and SCO0958 coding sequences were digested with $\mathrm{XbaI} / \mathrm{HindIII}$ restriction enzymes, and the resulting fragments were cloned into SpeI/ HindIII sites of pTR257, yielding plasmids pET28-0958FadD, pET28-0958-ACC, pET28-0958-FadR, pET280958-GpsA, and pET28-0958-0958, respectively.

\section{Total lipid analysis}

Batch cultures of $E$. coli strains were grown in LB media at $37^{\circ} \mathrm{C}$ until $\mathrm{OD}_{600 \mathrm{~nm}}$ was about 0.6. Then, protein expression was induced by addition of IPTG and/or L-arabinose. When the E. coli strain harbored pET28LPP $\beta$ and pBAD-0958 plasmids, L-arabinose was first added at $\mathrm{OD}_{600 \mathrm{~nm}} 0.6$ and IPTG was added $1 \mathrm{~h}$ later. After induction, the cultures were kept overnight at $23^{\circ} \mathrm{C}$ with agitation. For ${ }^{14} \mathrm{C}$ labeled experiments $3 \mu \mathrm{Ci}$ $\left[{ }^{14} \mathrm{C}\right]$-acetate $(58.9 \mathrm{Ci} / \mathrm{mol}$, Perkin Elmer) was added to $5 \mathrm{~mL}$ of culture, at the same time as the protein induction. Cultures were then normalized by CDW and total lipids were extracted as described by Bligh and Dyer [44].

The lipid extracts were dried and analyzed by TLC on silica gel 60 F254 plates $(0 \pm 2 \mathrm{~mm}$, Merck), using the solvent systems hexane/diethylether/acetic acid (75:25:1, v/v/v) [16]. Lipid fractions were visualized by $\mathrm{Cu}$-phosphoric staining and identified by comparing to the mobility of known standards. For ${ }^{14} \mathrm{C}$ labeled lipids the radioactivity incorporated into each lipid fraction was detected by autoradiography using Carestream ${ }^{\bullet}$ Kodak $^{\circ}$ BioMax $^{\oplus}$ MR films. All the TLC plates were digitalized and the spots were quantified using ImageJ v1.48 software.

\section{SDS-PAGE and immunoblot}

SDS-PAGE and immunoblot analyses using nitrocellulose membranes were carried out using standard protocols $[45,46]$. For detection of the His-tagged proteins, mouse monoclonal anti-His antibodies (QIAGEN ${ }^{\mathrm{m}}$ ) were used at a dilution of 1:1000. Anti-mouse IgG-alkaline phosphatase conjugates were used as secondary antibodies at a dilution of 1:3000. His-tagged proteins were visualized by immunoblots using chromogenic detection as described by the manufacturer.

\section{Triacylglycerol quantification by high resolution LC-MS}

For quantification of triacylglycerides, the biomass was lyophilized and weighed, and $1 \mathrm{mg}$ was processed for total lipid extraction as described by Bligh and Dyer [44]. $10 \mu \mathrm{g}$ of cetyl palmitate was added before extraction as an internal standard for load control. Then $500 \mu \mathrm{L}$ of chloroform were added to solubilize lipids and $50 \mu \mathrm{L}$ of the lipid extract were diluted in an equal part of methanol. $5 \mu \mathrm{L}$ of this solution were injected and separated on a ZORBAX Eclipse XDB-C8 column $(3.0 \times 50 \mathrm{~mm}$, particle size $=1.8 \mu \mathrm{m}$; Agilent, USA) using methanol supplemented with $5 \mathrm{mM}$ ammonium acetate as the mobile phase. The outlet of the liquid chromatograph was connected to a micrOTOF mass spectrometer (Bruker Daltonik, Bremen, Germany) operating in the positiveion mode, and the data was acquired online in the mass range $\mathrm{m} / \mathrm{z}$ 300-1500. TAGs were detected as ammonium adducts in the range of 6-20 min of the chromatography run. A calibration curve was done using pure 
tripalmitin as a standard. The TAG concentration in the samples was calculated by the linear regression equation obtained from the calibration curve.

\section{Fed-batch cultivation}

A BioFLO 1102 Lt Fermentor/Bioreactor (New Brunswik Scientific ${ }^{\circ}$ ) containing $0.9 \mathrm{~L}$ of minimal medium described above was autoclaved at $121^{\circ} \mathrm{C}$ for $30 \mathrm{~min}$. After sterilization, $50 \mathrm{mg} \mathrm{Km}, 20 \mathrm{mg} \mathrm{Cm}, 20$ g glucose, $0.5 \mathrm{~g} \mathrm{MgSO}_{4}$. $7 \mathrm{H}_{2} \mathrm{O}$, and $1.5 \mathrm{~mL}$ trace element solution were added aseptically to the bioreactor. The reactor temperature, $\mathrm{pH}$, and dissolved oxygen $\left(\mathrm{DO}_{2}\right)$ were monitored using specific probes (Mettler-Toledo ${ }^{\circ}$ ). The temperature was maintained at $37^{\circ} \mathrm{C}$ or $23^{\circ} \mathrm{C}$ using a heat blanket and cooling water. The reactor $\mathrm{pH}$ was maintained at $7.0 \pm 0.1$ by the addition of $15 \%(\mathrm{v} / \mathrm{v}) \mathrm{NH}_{4} \mathrm{OH}$. When the ammonium concentration reached $200 \mathrm{mM}, 15 \% \mathrm{NH}_{4} \mathrm{OH}$ was replaced by $5 \mathrm{M} \mathrm{NaOH}$. Agitation was provided by a double sixbladed impeller with the stir speed set at $700 \mathrm{rpm}$, and the gas flow was alternated between air and oxygen to ensure that the $\mathrm{DO}_{2}$ content did not decrease below $40 \%$ saturation. The gas inflow rate was maintained at $0.33 \mathrm{vvm}$. Foaming was controlled by the addition of Antifoam A (Code A5633, Sigma-Aldrich ${ }^{\circ}$. The glucose and ammonium concentrations were measured with enzymatic kits from Wiener $\mathrm{Lab}^{\circ}$, codes 1400101 and 1810050, respectively. Inoculation was carried out at $37^{\circ} \mathrm{C}$ with $100 \mathrm{~mL}$ of seed culture at $\mathrm{OD}_{600 \mathrm{~nm}}$ approximately 2 . When glucose in the initial medium was depleted at $12 \mathrm{~h}$ after inoculation $\left(\mathrm{OD}_{600 \mathrm{~nm}}\right.$ about 20$)$, a nutrient feed consisting of the described carbon source solution was initiated at a fixed rate of $0.2 \mathrm{~mL} / \mathrm{min}$. When the culture reached $\mathrm{OD}_{600 \mathrm{~nm}}$ about $60,16 \mathrm{~h}$ after inoculation, the temperature was reduced to $23^{\circ} \mathrm{C}$, the nutrient feed rate was shifted to a fixed rate of $0.1 \mathrm{~mL} / \mathrm{min}$, and heterologous protein expression was induced by the addition of $100 \mu \mathrm{M}$ IPTG and $0.1 \%$ $(w / v)$ L-arabinose. The culture was allowed to continue until maximum TAG production was reached $(68.5 \mathrm{~h})$. TAG production (mg TAG/L), at a given time point, was calculated from the TAG content of the corresponding sample, considering the current $\mathrm{OD}_{600 \mathrm{~nm}}$ and volume of the sample, and the current volume of the fed-batch culture at this time point.

\section{Additional file}

Additional file 1: Figure S1. Western blot analysis of Sco0958 and $L p p \beta$ in the strains described in Figure 3. Protein extracts were resolved by SDS-PAGE in a $12 \%$ polyacrylamide gel and detected by immunoblotting using anti-His antibodies as described in the Methods section. Lane 1, MPS11; lanes 2 and 3, MPS11/pBAD-0958/pET28-LPP $\beta$; lanes 4 and 5, MPS11/pET28-0958/pBAD-LPPß; lane 6, MPS11/pET28-0958-LPPß; lane 7 , MPS11/pBAD-0958-Lppß. Table S1. Cell TAG content of different E. coli strains determined by high resolution LC-MS. Table S2. E. coli triglyceride fatty acid relative composition.

\section{Abbreviations}

Ara: arabinose; CDW: cell dry weight; DAG: diacylglycerol;

DGAT: diacylglycerol acyltransferase; FA: fatty acid; IPTG: isopropyl- $\beta-D-$ thiogalactopyranoside; LC-MS: liquid chromatography coupled to mass spectrometry; OD: optical density; PAP: phosphatidic acid phosphatase; PL: phospholipid; TAG: triacylglycerol, triglyceride; TLC: thin layer chromatography.

\section{Competing interests}

The authors declare that they have no competing interests.

\section{Authors contributions}

SC, MS, SMB, AA, and HG designed all the experiments. SC and MS constructed the plasmid backbones. SC, MS, and SMB performed all the experiments. SC, AA, and HG wrote the manuscript. All the authors read and approved the final manuscript.

\section{Acknowledgements}

We thank G. Millán for LC-MS technical assistance. This work was supported by ANPCyT grants PICT 2012-0168 to HG and PICT 2011-2005 to AA. HG and AA are members of the Research Career, and SMB, SC, and MS are doctoral fellows of CONICET.

Received: 5 September 2014 Accepted: 18 November 2014

Published online: 24 December 2014

\section{References}

1. Alvarez HM, Steinbuchel A: Triacylglycerols in prokaryotic microorganisms. Appl Microbiol Biotechnol 2002, 60:367-376.

2. Comba S, Arabolaza A, Gramajo H: Emerging engineering principles for yield improvement in microbial cell design. Comput Struct Biotechnol J 2012, 3:e201210016.

3. Kalscheuer R, Stoveken T, Luftmann H, Malkus U, Reichelt R, Steinbuchel A: Neutral lipid biosynthesis in engineered Escherichia coli: jojoba oil-like wax esters and fatty acid butyl esters. Appl Environ Microbiol 2006, 72:1373-1379.

4. Choi YJ, Lee SY: Microbial production of short-chain alkanes. Nature 2013, 502:571-574.

5. Peralta-Yahya PP, Zhang F, del Cardayre SB, Keasling JD: Microbial engineering for the production of advanced biofuels. Nature 2012, 488:320-328.

6. Liang MH, Jiang JG: Advancing oleaginous microorganisms to produce lipid via metabolic engineering technology. Prog Lipid Res 2013, 52:395-408.

7. Kurosawa K, Boccazzi P, de Almeida NM, Sinskey AJ: High-cell-density batch fermentation of Rhodococcus opacus PD630 using a high glucose concentration for triacylglycerol production. J Biotechnol 2010, 147:212-218.

8. Rucker J, Paul J, Pfeifer BA, Lee K: Engineering E. coli for triglyceride accumulation through native and heterologous metabolic reactions. Appl Microbiol Biotechnol 2013, 97:2753-2759.

9. Janssen HJ, Steinbuchel A: Fatty acid synthesis in Escherichia coli and its applications towards the production of fatty acid based biofuels. Biotechnol Biofuels 2014, 7:7.

10. Lin F, Chen Y, Levine R, Lee K, Yuan Y, Lin XN: Improving fatty acid availability for bio-hydrocarbon production in Escherichia coli by metabolic engineering. PLoS One 2013, 8:e78595.

11. Janssen HJ, Steinbuchel A: Production of triacylglycerols in Escherichia coli by deletion of the diacylglycerol kinase gene and heterologous overexpression of atfA from Acinetobacter baylyi $\mathrm{ADP}_{1}$. Appl Microbiol Biotechnol 2014, 98:1913-1924.

12. Comba S, Menendez-Bravo S, Arabolaza A, Gramajo H: Identification and physiological characterization of phosphatidic acid phosphatase enzymes involved in triacylglycerol biosynthesis in Streptomyces coelicolor. Microb Cell Fact 2013, 12:9.

13. Raetz CR, Newman KF: Neutral lipid accumulation in the membranes of Escherichia coli mutants lacking diglyceride kinase. J Biol Chem 1978, 253:3882-3887.

14. Raetz CR, Newman KF: Diglyceride kinase mutants of Escherichia coli: inner membrane association of 1,2-diglyceride and its relation to synthesis of membrane-derived oligosaccharides. J Bacteriol 1979, 137:860-868. 
15. Arabolaza A, Rodriguez E, Altabe $\mathrm{S}$, Alvarez H, Gramajo H: Multiple pathways for triacylglycerol biosynthesis in Streptomyces coelicolor. Appl Environ Microbiol 2008, 74:2573-2582

16. Rotering $\mathrm{H}$, Raetz CR: Appearance of monoglyceride and triglyceride in the cell envelope of Escherichia coli mutants defective in diglyceride kinase. J Biol Chem 1983, 258:8068-8073.

17. Dillon DA, Wu WI, Riedel B, Wissing JB, Dowhan W, Carman GM: The Escherichia coli pgpB gene encodes for a diacylglycerol pyrophosphate phosphatase activity. J Biol Chem 1996, 271:30548-30553.

18. Touze T, Blanot D, Mengin-Lecreulx D: Substrate specificity and membrane topology of Escherichia coli PgpB, an undecaprenyl pyrophosphate phosphatase. J Biol Chem 2008, 283:16573-16583.

19. Lu YH, Guan Z, Zhao J, Raetz CR: Three phosphatidylglycerol-phosphate phosphatases in the inner membrane of Escherichia coli. J Biol Chem 2011, 286:5506-5518.

20. Kalscheuer R, Steinbuchel A: A novel bifunctional wax ester synthase/ acyl-CoA:diacylglycerol acyltransferase mediates wax ester and triacylglycerol biosynthesis in Acinetobacter calcoaceticus ADP1. J Biol Chem 2003, 278:8075-8082

21. Kalscheuer R, Stolting T, Steinbuchel A: Microdiesel: Escherichia coli engineered for fuel production. Microbiology 2006, 152:2529-2536.

22. Black PN, DiRusso CC, Metzger AK, Heimert TL: Cloning, sequencing, and expression of the fadD gene of Escherichia coli encoding acyl coenzyme A synthetase. J Biol Chem 1992, 267:25513-25520.

23. Campbell JW, Cronan JE Jr: The enigmatic Escherichia coli fadE gene is yafH. J Bacteriol 2002, 184:3759-3764.

24. Bao X, Ohlrogge J: Supply of fatty acid is one limiting factor in the accumulation of triacylglycerol in developing embryos. Plant Physiol 1999, 120:1057-1062.

25. Cronan JE Jr, Subrahmanyam S: FadR, transcriptional co-ordination of metabolic expediency. Mol Microbiol 1998, 29:937-943.

26. Campbell JW, Cronan JE Jr: Escherichia coli FadR positively regulates transcription of the $f a b B$ fatty acid biosynthetic gene. J Bacteriol 2001 183:5982-5990.

27. Zhang F, Ouellet M, Batth TS, Adams PD, Petzold CJ, Mukhopadhyay A, Keasling JD: Enhancing fatty acid production by the expression of the regulatory transcription factor FadR. Metab Eng 2012, 14:653-660.

28. Rodriguez E, Banchio C, Diacovich L, Bibb MJ, Gramajo H: Role of an essential acyl coenzyme A carboxylase in the primary and secondary metabolism of Streptomyces coelicolor A3(2). Appl Environ Microbiol 2001, 67:4166-4176

29. Gago G, Diacovich L, Arabolaza A, Tsai SC, Gramajo H: Fatty acid biosynthesis in actinomycetes. FEMS Microbiol Rev 2011, 35:475-497.

30. Steen EJ, Kang Y, Bokinsky G, Hu Z, Schirmer A, McClure A, Del Cardayre SB, Keasling JD: Microbial production of fatty-acid-derived fuels and chemicals from plant biomass. Nature 2010, 463:559-562.

31. Schirmer A, Rude MA, Li X, Popova E, del Cardayre SB: Microbial biosynthesis of alkanes. Science 2010, 329:559-562.

32. Lennen RM, Braden DJ, West RA, Dumesic JA, Pfleger BF: A process for microbial hydrocarbon synthesis: overproduction of fatty acids in Escherichia coli and catalytic conversion to alkanes. Biotechnol Bioeng 2010, 106:193-202

33. Liu T, Vora $H$, Khosla C: Quantitative analysis and engineering of fatty acid biosynthesis in E. coli. Metab Eng 2010, 12:378-386.

34. Hofvander $\mathrm{P}$, Doan TTP, Hamberg M: A prokaryotic acyl-CoA reductase performing reduction of fatty acyl-CoA to fatty alcohol. FEBS Lett 2011, 585:3538-3543.

35. Zheng YN, Li LL, Liu Q, Yang JM, Wang XW, Liu W, Xu X, Liu H, Zhao G, Xian M: Optimization of fatty alcohol biosynthesis pathway for selectively enhanced production of C12/14 and C16/18 fatty alcohols in engineered Escherichia coli. Microb Cell Fact 2012, 11:65

36. Akhtar MK, Turner NJ, Jones PR: Carboxylic acid reductase is a versatile enzyme for the conversion of fatty acids into fuels and chemical commodities. Proc Natl Acad Sci 2013, 110:87-92.

37. Torella JP, Ford TJ, Kim SN, Chen AM, Way JC, Silver PA: Tailored fatty acid synthesis via dynamic control of fatty acid elongation. Proc Natl Acad SCi U S A 2013, 110:11290-11295.

38. Baba T, Ara T, Hasegawa M, Takai Y, Okumura Y, Baba M, Datsenko KA, Tomita M, Wanner BL, Mori H: Construction of Escherichia coli K-12 in-frame, single-gene knockout mutants: the Keio collection. Mol Syst Biol 2006, 2:2006.0008
39. Datsenko KA, Wanner BL: One-step inactivation of chromosomal genes in Escherichia coli K-12 using PCR products. Proc Natl Acad Sci U S A 2000, 97:6640-6645

40. Hanahan D: Studies on transformation of Escherichia coli with plasmids. J Mol Biol 1983, 166:557-580.

41. Guzman LM, Belin D, Carson MJ, Beckwith J: Tight regulation, modulation and high-level expression by vectors containing the arabinose PBAD promoter. J Bacteriol 1995, 177:4121-4130.

42. Cherepanov PP, Wackernagel W: Gene disruption in Escherichia coli: TcR and $\mathrm{KmR}$ cassettes with the option of Flp-catalyzed excision of the antibiotic-resistance determinant. Gene 1995, 158:9-14.

43. Menendez-Bravo S, Comba S, Sabatini M, Arabolaza A, Gramajo H: Expanding the chemical diversity of natural esters by engineering a polyketide-derived pathway into Escherichia coli. Metab Eng 2014, 24:97-106

44. Bligh EG, Dyer WJ: A rapid method of total lipid extraction and purification. Can J Biochem Physiol 1959, 37:911-917.

45. Laemmli UK: Cleavage of structural proteins during the assembly of the head of bacteriophage T4. Nature 1970, 227:680-685.

46. Sambrock J, Fritsch FEF, Maniatis T: Molecular Cloning: A Laboratory Manual. Cold Spring Harbor, NY: Cold Spring Harbor Laboratory Press; 1989.

\section{Submit your next manuscript to BioMed Central and take full advantage of:}

- Convenient online submission

- Thorough peer review

- No space constraints or color figure charges

- Immediate publication on acceptance

- Inclusion in PubMed, CAS, Scopus and Google Scholar

- Research which is freely available for redistribution
C BioMed Central 